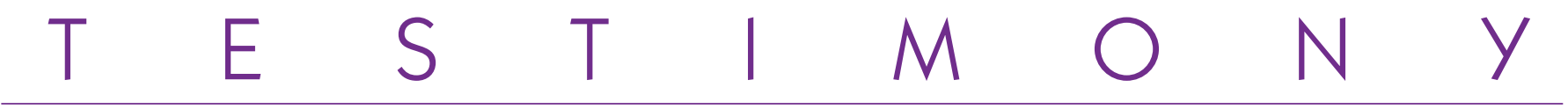

\title{
About my case
}

\author{
M. El Okeily \\ Maxillofacial surgeon, Private practice
}

\begin{abstract}
Patient care in orthognathic surgery is not easy. It is important to be aware of a number of diverse parameters that affect the patients and their families. These parameters can be technical, functional, and of course psychological. To us practitioners, the decisions often seem clear but it is difficult to put yourself in a patient's place to understand the hardships they endure on their "orthognathic journey." To this end, what could be better than actually assuming the role of a patient in need of orthognathic surgery. Experiencing surgery as a patient has certainly changed my views, whereby I feel more closely linked to my patients and to their experience of the different treatment phases.
\end{abstract}

\section{The Motivation}

It is difficult to remember exactly when you became aware of the problem. In adolescence, this "jaw problem" was certainly present but it was understated and I pushed it to the back of my mind.
I was resigned to accept my fate and live the way nature had made me. I cannot put myself in the position of a teenager who is being guided by their parents because that was not the case with me. However, I can speak as an adult who can make their own decisions. The fact that I myselfam a practitionerwho on a daily basis deals with patients seeking orthognathic surgery served to jumpstart my process. I needed to understand on some level the discussions that I have on a daily basis with my patients. Even if intellectually this process seems logical, in the medical world, it is far from an obvious choice. Simply consider the number of cardiologists, vascular surgeons, oncologists, and even tobaccologists who smoke! In my case, I had several reasons among which were; the existence of periodontal risk, dysfunction of the temporomandibular joints, and the presence of postural pains.

Concerning the periodontal risk, I have a family history and I have early traces of gingival recession of the mandibular incisive canal. Faced with a clinical 
situation, I needed to make a choice, but this time as a patient. I researched a bit on evidencebased science, and so I seized the opportunity however minimal to delay or avoid any loosening. I always experienced a painless cracking of the temporomandibular articulations (TMA) together with a sigmoid notch and disc protrusion. Nonetheless, it was when I experienced an episode of painful, temporary blockage that I was alerted to the seriousness of having dysfunctional TMAs. The restricted mouth opening that usually results is so painful that even if it only lasts for a few days, it is definitely not something to be ignored.

Finally, with regard to postural pains, I also had cervical symptomology with recurring pains and frequent episodes of neck stiffness (one every month and a half). I had also experienced two lumbosciatic episodes.

\section{Making the Decision}

Once sufficiently motivated, all that remained was to act. For me, two parameters were very important. It is probably the fact that I was approaching 40 years of age and I exhibited a constant, general concern about my body image (on top of everything I was training for my first marathon), which motivated me to seek surgicalorthodontic treatment.

\section{Starting Treatment}

When you have a busy work schedule, you need to plan the treatment procedure specifically so that it has minimum impact on your personal and professional life. I wanted the intervention to take place at the end of December so that it would not affect my work. Then you need to choose the surgical team. It is a very important choice because you are entrusting your body to practitioners in whom you must have complete confidence. I have the advantage of knowing how my colleagues work. The treatment stages were set during the initial assessment.

I had a class-II retromandibular vein, as well as a transverse deficiency on the hypodivergent facial types. The maxilla was generally well-positioned. I hoped, therefore, to be treated by with mandibular advancement, which gave me the impression of the treatment process being easy.

\section{Orthodontics}

When the orthodontic device had been placed on I prepared myself to respond to the eventual questions from my patients and from my family about why I was having treatment. In actuality, there were very few questions. Although I was wearing a standard vestibular apparatus, few patients noticed; when they did, the comments were rather positive. In my family circle too, there were few comments.

I genuinely found orthodontic surgery to be less painful than I had imagined. There was some pain in the first 24-48 hours after an arch change, but this eased up once I took a paracetamol tablet. On the other hand, the upkeep of the apparatus and the 
discomfort during meals were sometimes difficult to bear.

\section{Surgery}

Generally, patients are apprehensive regarding surgical intervention. Personally, I was confident, impatient and in a hurry to be operated on. I knew the importance of having the right mindset immediately after the surgery. I had a quiet dinner with my orthodontist on the eve of the surgery. I remember falling asleep and waking up a few hours after the surgery. At this point, my expectations were worse than the reality. The edema was annoying but there was no pain. I understood that I needed time to regain my normal facial functions and sensations. I was happy to feel, the paresthesia indicative of the recuperation of the nerves, from right to left. I have now regained $100 \%$ right and 99\% left as I experience some tingling in a small area from time to time.

I also discovered that eating can be different in a way that I never thought of. For example, some food items like (foie gras) could be absorbed by letting them melt in the mouth without having to chew.

\section{FROM THEIR POINT OF VIEW}

\section{The Orthodontist Relates Their Experience}

Most of us are apprehensive about having to care for our family members or colleagues. A well-known adage says that in such a situation, treatment does not go as planned. When I undertook orthodontic surgery for one
The same evening of the surgery, I was responding to patients' emails, and 3 weeks after the surgery, I attended a conference on cosmetic surgery.

The only two annoying factors are the abstinence from sport and, even more so, the placement of the tongue in its new environment. I never had lingual dysfunction before the intervention. Yes, it took 2-3 months to find the correct position when my tongue is at rest, when swallowing, and especially when speaking. For this, I am thankful for the excellent maxillofacial kinesiotherapy specialist, who has guided me well throughout this process.

\section{Post Treatment}

As the final stage of orthodontic intervention progressed I observed:

- A spontaneous decrease in gingival recession;

- A buccal aperture without any TMArelated protrusions;

- No more neck stiffness for 2 years.

On the other hand, my family took a few months to get used to my new profile, constantly asking me: "are you sure it did not go too far?"

of my best friends, who is a maxillofacial surgeon and whose specialty is orthognathic surgery, I was not without my doubts, on a professional and personal level. Will they be a good patient? How will our friendship change should the surgery fail?

Through this experience, I was made aware of the power behind the 
patient-therapist relationship. We sit in the armchair and we tend to ignore all these associated parameters. The treatment progressed just as it would have with any other patient.

What is more, our personal relationship has allowed me to see my friend and patient evolve in their domestic life. I have seen how much a person's family can question the legitimacy of orthodontic surgery. After this experience, I now work with the patient to address all the questions posed by their family and the related queries.

Conflict of interest: The author declares that they have no conflict of interest. 